\title{
The Influence of Patient Characteristics on Anticholinergic Events in Older People
}

\author{
Mohammed Saji Salahudeen Prasad S. Nishtala Stephen B. Duffull \\ School of Pharmacy, University of Otago, Dunedin, New Zealand
}

\section{Key Words}

Anticholinergic events · Risk factors · Adverse events · Elderly · Older people ·

Antimuscarinic response $\cdot$ Anticholinergic burden

\begin{abstract}
Aims: To examine patient characteristics that predict adverse anticholinergic-type events in older people. Methods: This retrospective population-level study included 2,248 hospitalised patients. Individual data on medicines that are commonly associated with anticholinergic events (delirium, constipation and urinary retention) were identified. Patient characteristics examined were medicines with anticholinergic effects (ACh burden), age, sex, non-anticholinergic medicines (non-ACM), Charlson comorbidity index scores and ethnicity. The Akaike information criterion was used for model selection. The data were analysed using logistic regression models for anticholinergic events using the software NONMEM. Results: ACh burden was found to be a significant independent predictor for developing an anticholinergic event [adjusted odds ratio (aOR): 3.21, 95\% CI: 1.23-5.81] for those taking an average of 5 anticholinergic medicines compared to those taking 1 . Both non-ACM and age were also independent risk factors (aOR: 1.41, 95\% CI: 1.31-1.51 and aOR: 1.08, 95\% CI: 1.05-1.10, respectively). Conclusion: To our knowledge, this is the first study that has examined population-level data in a nonlinear model framework to predict anticholinergic-type adverse events. This study evaluated the relationship between important patient characteristics and the occurrence of anticholinergic-type events. These findings reinforce the clinical significance of reviewing anticholinergic medicines in older people.

(C) 2016 The Author(s)

Published by S. Karger AG, Basel
\end{abstract}

\section{Introduction}

Older people taking medicines with anticholinergic properties are at an increased risk of experiencing adverse events [1-6]. Anticholinergic medicines bind to the muscarinic receptors, and the decreased cholinergic neurotransmission impacts both peripheral and 
Salahudeen et al.: The Influence of Patient Characteristics on Anticholinergic Events in Older People

central body systems [3, 7-10]. Peripheral anticholinergic adverse effects mainly include constipation, urinary retention, dry mouth, blurred vision, peristaltic reduction and tachycardia. The central anticholinergic adverse effects of clinical interest are cognitive impairment, confusion and delirium [11-14]. The risk of anticholinergic adverse events has been found to increase with age, pre-existing cognitive impairment, polypharmacy, comorbidities and cholinergic neuronal degeneration, and it is influenced by individual pharmacokinetic and pharmacodynamic variability $[2,8,10,15,16]$.

The frequency of adverse drug reactions reported in older adults ( $\geq 65$ years) is 7 -fold higher than in younger adults [17]. Epidemiological studies have reported that $20-50 \%$ of the older population are routinely exposed to medicines that possess anticholinergic properties $[18,19]$. Recent literature has highlighted that up to $40 \%$ of all institutionalised older people take medicines with known anticholinergic activity $[2,20]$. Similarly, it has been reported that among hospitalised older people, the prevalence of those taking anticholinergic medicines increased significantly ( $7.2 \pm 3.5$ vs. $5.7 \pm 3.1 ; p<0.001$ ) during their hospital stay [21].

The cumulative exposure to multiple medicines with anticholinergic properties is widely referred to as anticholinergic $(\mathrm{ACh})$ burden $[10,12]$. Numerous studies have reported ACh burden to be an important predictor of cognitive and physical impairment in older populations [1, 15, 22-26]. A higher ACh burden is associated with greater risks of morbidity and mortality, a longer length of hospital stay, institutionalisation and functional and cognitive decline in older people $[3,7-9,12,27-34]$. Reducing the ACh burden can cause significant improvements in short-term memory, delirium, behaviour, daily-living and quality of life [7, $15,28,35]$. Our recent systematic review identified two cohort studies [36, 37] that demonstrated an improvement of cognitive performance after the discontinuation of anticholinergic medicines [10]. A retrospective study by Nishtala et al. [15] found that a significant interaction exists between age $\geq 65$ years and the exposure to medicines with anticholinergic properties [15]. In their study, Hilmer et al. [28] found a relationship between higher anticholinergic exposure and impaired physical function after adjusting for age, sex, race and comorbidities.

The current model assumes that the antimuscarinic response contributed by each drug is linear and additive [28]. However, there is a need to further explore the effect of anticholinergic events using nonlinear models which may reflect receptor binding more closely. Studies involving population-level data examining the exposure to anticholinergic drugs and adverse events are limited. Furthermore, clinical evidence implicating anticholinergic adverse events and the influence of associated patient characteristics in a nonlinear framework is lacking. To provide additional insights into the relationship between anticholinergic drugs and the influence of patient characteristics, we proposed a model-based approach to predict anticholinergic-type adverse events (central and peripheral adverse events) in this study. The main objective of this study was to explore the relationship between effects of important patient characteristics and the occurrence of anticholinergic-type events.

\section{Methods}

\section{Data Source and Study Population}

This retrospective population-level study included hospitalised individuals in 2010 identified from the national health data collections maintained by the Ministry of Health of New Zealand [38, 39]. Pharmaceutical collections are used by the Pharmaceutical Management Agency (PHARMAC) and the Ministry of Health of New Zealand to administer payment to pharmacists for dispensing medicines that are publicly funded under the national medicines budget managed by PHARMAC. Data on dispensed medicines were obtained from the phar- 
Salahudeen et al.: The Influence of Patient Characteristics on Anticholinergic Events in Older People

maceutical collections for 2010. Demographic characteristics such as sex, date of birth, ethnicity and district health board of domicile were also extracted from the pharmaceutical collections. Patient information from the pharmaceutical collections was matched with hospital event data from the National Minimum Dataset using unique encrypted National Health Index numbers. We captured all medicines dispensed 14 days prior to the date of hospital admission to identify possible associations with outcomes of interest. Diagnoses in the National Minimum Dataset are coded using the International Classification of Diseases and Related Health Problems, 10th revision, Australian modification (ICD-10-AM). We identified ICD-10 codes for delirium (F05), constipation (K59) and urinary retention (R32, R39) from the coded diagnosis to capture anticholinergic events.

Overall, all hospitalised individuals aged $\geq 65$ years living in New Zealand who were receiving at least one prescription medicine during the period starting on January 1, 2010 to December 31, 2010 were included in this study. Individuals with missing data on one or a combination of the following variables were excluded from the dataset: age, sex, medicine and medicine strength/dose. In this study, delirium, constipation and urinary retention were examined for anticholinergic adverse effects. Delirium is the most common cause of acute cognitive dysfunction in hospitalised older people, and it is mediated by the antagonism of centrally acting muscarinic receptors $[40,41]$. These adverse effects were chosen because they reflect typical adverse pharmacological effects linked to central or peripheral muscarinic receptors associated with drug properties. Several studies have highlighted constipation and urinary retention as frequently occurring adverse anticholinergic effects in older people $[7,42,43]$.

A validated weighted comorbidity index [Charlson comorbidity index (CCI)] was used for the measurement of comorbidity burden [44]. It provides a weighted score of a subject's comorbidities which can be used to predict short- and long-term outcomes. Compared to other comorbidity indices, the CCI score can be assigned to the majority of patients and is readily available. Studies have shown that the CCI score predicts hospitalisation, adverse outcomes as well as mortality [45-47]. The extracted data were rearranged into NONMEM version 7.2 with the G95 FORTRAN compiler-readable format using MATLAB version R2013b.

Approval to undertake this study was provided by the Human Ethics Committee of the University of Otago, New Zealand (approval No. HD14/10).

\section{Exposure to Anticholinergic Medicines}

Numerous medicines possess anticholinergic activity, but many of them are unlikely to cause significant clinical symptoms when given as monotherapy [48]. Our study focused on the use of medicines with relevant central and peripheral anticholinergic effects. Hence, the study considered medicines that have been identified as carrying a mild, moderate or severe risk of anticholinergic properties. Anticholinergic exposure was defined based on a list of medicines with known anticholinergic activity from the expert-opinion rating scales [49]. From this exposure, ACh burden was defined in this study as the sum of all anticholinergic medicine(s) taken by an individual.

\section{Patient Characteristics and Modelling}

The dataset comprised various patient characteristics such as medicines with anticholinergic properties (ACh burden), age, sex, non-anticholinergic medicines (non-ACM), CCI, ethnicity and number of hospital admissions during the study period. In this study, the total of all medicines is a continuous variable equivalent to the sum of all non-ACMs and the number of all anticholinergic medicine(s) (ACh burden). We were interested in ACh burden, and hence defined the other part of the total number of medicines as non-ACM. The study considered both linear and nonlinear logit models to evaluate the patient characteristics. The linear 
Salahudeen et al.: The Influence of Patient Characteristics on Anticholinergic Events in Older People

models were constructed based on a stepwise forward addition of patient characteristics from the structural model to define the maximum number of important characteristics. The nonlinear model building was performed by adding patient characteristics into three different locations to predict various effects. In all models, second-level interactions were considered for testing variables of different patient characteristics.

Additional exploration was possible with the nonlinear model, which resembles a pharmacological binding model (which consider drug(s) binding to the receptor), and patient characteristics could be incorporated to test whether they (1) increased the risk of events independent of ACh burden (i.e. they pose a risk even in the absence of ACh burden) by adding patient characteristics to the baseline $\left(\beta_{0}\right),(2)$ increased the maximal anticholinergic effect of ACh burden (i.e. an overall greater effect is seen with ACh burden) by adding an interaction of patient characteristics on Emax or (3) increased the apparent potency of the ACh burden (i.e. greater effects were seen for a given ACh burden value) by adding a patient characteristics on $\mathrm{ACH}_{50}$ that provides $50 \%$ of the maximal effect. The following logistic models where used to describe the linear and nonlinear predictors:

Linear Framework

$$
\log _{e}\left\{\frac{\pi}{1-\pi}\right\}=\beta_{0}+\beta_{1} X_{1}+\ldots+\beta_{n} X_{n}
$$

where,

$$
\log _{e}\left\{\frac{\pi}{1-\pi}\right\}
$$

represents the log odds (logit) for reporting adverse anticholinergic effects, $X_{n}$ represents the presence or absence of the $n$-th variable, $\beta_{n}$ represents the parameter estimate and $\beta_{0}$ represents the intercept (base probability).

Nonlinear Framework

$$
\log _{e}\left\{\frac{\pi}{1-\pi}\right\}=\beta_{0}+\text { Emax } \frac{\text { ACh burden }}{\left(A C H_{50}+\text { ACh burden }\right)} .
$$

Here, Emax is the maximal effect that can be achieved, and $\mathrm{ACH}_{50}$ is the $\mathrm{ACh}$ burden that provides $50 \%$ of the maximal effect. In this modelling framework, we can consider the influence of patient characteristics on $\beta_{0}$ (the intercept), $E \max$ (the maximal effect) and $\mathrm{ACH}_{50}$ (the apparent ACh burden potency).

All logit expressions were transformed using the expit function $\left(e^{x} /\left(1+e^{x}\right)\right)$ for a standard logistic regression. Model building for the linear and nonlinear frameworks was carried out from the base model (a model that considered intercept only).

\section{Statistical Analysis}

The data were analysed using logistic regression (in this case expanded to generalised nonlinear models) for all three anticholinergic events (delirium, constipation and urinary retention) using the software NONMEM (version 7.2). The NONMEM (nonlinear mixed effects modelling) software is a regression program that can accommodate repeated measurement data, where each individual may contribute multiple observations. Since some individuals contributed to more than one occasion (number of hospital admissions), the subject-specific 
Table 1. Characteristics of the study population

\begin{tabular}{|c|c|c|c|c|c|}
\hline & $\begin{array}{l}\text { Total dataset } \\
\text { population }\end{array}$ & Delirium & Constipation & $\begin{array}{l}\text { Urinary } \\
\text { retention }\end{array}$ & $\begin{array}{l}\text { For total } \\
\text { events }\end{array}$ \\
\hline Patients & 2,248 & 48 & 80 & 20 & - \\
\hline Total events & $148(6.6)$ & $48(2.1)$ & $80(3.6)$ & $20(0.9)$ & $148(6.6)$ \\
\hline Male & $1,020(45.4)$ & $17(35.4)$ & 27 (33.8) & $12(60.0)$ & $56(37.9)$ \\
\hline Female & $1,228(54.6)$ & $31(64.6)$ & $53(66.2)$ & $8(40.0)$ & $92(62.1)$ \\
\hline Age, years ${ }^{\mathrm{a}}$ & $79 \pm 8.0$ & $85 \pm 6.7$ & $82 \pm 8.6$ & 849.6 & $84 \pm 8.3$ \\
\hline Total medicines & $3.48 \pm 2.5$ & $5.88 \pm 2.8$ & $5.89 \pm 3.0$ & $5.45 \pm 2.5$ & $5.74 \pm 2.8$ \\
\hline Occasions $^{\mathrm{b}}$ & $1.10 \pm 0.4$ & $1.08 \pm 0.3$ & $1.05 \pm 0.2$ & $1.00 \pm 0.0$ & $1.04 \pm 0.2$ \\
\hline $\mathrm{CCI}$ & $1.10 \pm 0.3$ & $1.42 \pm 1.5$ & $1.16 \pm 1.4$ & $1.10 \pm 1.3$ & $1.23 \pm 1.4$ \\
\hline \multicolumn{6}{|l|}{ Ethnicity } \\
\hline European & $1,855(82.5)$ & $46(95.8)$ & $64(80.0)$ & $17(85.0)$ & $127(85.8)$ \\
\hline Māori & $158(7.0)$ & $2(4.2)$ & $8(10.0)$ & $2(10.0)$ & $12(8.1)$ \\
\hline Pacific & $109(4.8)$ & 0 & $1(1.3)$ & 0 & $1(0.7)$ \\
\hline Asian & $82(3.6)$ & 0 & $6(7.5)$ & $1(5.0)$ & $7(4.7)$ \\
\hline
\end{tabular}

random effects were also considered. In this framework, a second-order conditional estimation method (the Laplacian option) was used in NONMEM.

Patient characteristics with a statistically significant effect were incorporated in the model, which significantly reduced the unexplained between-subject variability (in circumstances where this was estimated), and the Akaike information criterion was used in this study for model selection [50]. Continuous data from the patient characteristics (such as age) were normalised in the model by the mean population value to derive a stable conversion of data with less variance. The analysis continued with the stepwise forward addition of patient characteristics from the structural base model to define the maximum number of important patient characteristics. The structural model was then revised by considering interaction terms to provide the final model.

The $95 \%$ confidence interval for the adjusted odds ratio (aOR) values was estimated using a parametric bootstrap in which 10,000 parameter values were simulated in MATLAB under the posterior distribution from the NONMEM parameter estimates. The confidence interval was then taken as the 2.5th and 97.5th percentiles of the simulations.

\section{Results}

The study sample consisted of 2,248 older hospitalised individuals. The incidence rates of anticholinergic-type adverse events in the dataset for delirium, constipation and urinary retention were $2.1,3.6$ and $0.9 \%$, respectively. The mean age ( \pm SD) of the study population was $79 \pm 8$ years. A detailed summary of the patient characteristics is given in table 1. The overall incidence rate of all events in the dataset was $6.6 \%$, and the baseline estimate from logistic regression estimated an average incidence rate of the three events of $2.2 \%$. However, considering the Akaike information criterion, the complexity of the multivariate model, the biological plausibility and the pharmacological mechanism, the nonlinear model was preferred over the linear model. A detailed summary of both the linear 
Table 2. Particulars of the anticholinergic medications identified from the study population $(n=69)$

\begin{tabular}{|c|c|c|c|}
\hline System & Therapeutic class & $\begin{array}{l}\text { Medicines with anticholinergic } \\
\text { activity }\end{array}$ & Frequency \\
\hline \multirow[t]{8}{*}{ Cardiovascular system } & Diuretic & $\begin{array}{l}\text { furosemide }(\mathrm{H}, \mathrm{L}) \\
\text { chlortalidone }(\mathrm{L})\end{array}$ & $\begin{array}{r}981 \\
6 \\
\end{array}$ \\
\hline & Beta-blocker & $\begin{array}{l}\text { metoprolol (L) } \\
\text { atenolol (L) }\end{array}$ & $\begin{array}{r}939 \\
63\end{array}$ \\
\hline & Nitrate & isosorbide (L) & 237 \\
\hline & Antiarrhythmic & $\operatorname{digoxin}(\mathrm{H}, \mathrm{L})$ & 309 \\
\hline & Calcium channel blocker & $\begin{array}{l}\text { diltiazem (L) } \\
\text { nifedipine (L) }\end{array}$ & $\begin{array}{r}216 \\
9\end{array}$ \\
\hline & Platelet inhibitor & dipyridamole (L) & 147 \\
\hline & ACE inhibitor & captopril (L) & 3 \\
\hline & Anticoagulant & warfarin $(\mathrm{L})$ & 477 \\
\hline Respiratory system & Xanthine & theophylline (M, L) & 6 \\
\hline \multirow[t]{32}{*}{ Neurological system } & \multirow[t]{3}{*}{ SSRI } & citalopram (L) & 486 \\
\hline & & fluoxetine (L) & 114 \\
\hline & & paroxetine $(\mathrm{H}, \mathrm{M}, \mathrm{L})$ & 78 \\
\hline & SNRI & venlafaxine (L) & 21 \\
\hline & \multirow{8}{*}{ Antidepressant } & amitriptyline $(\mathrm{H})$ & 414 \\
\hline & & imipramine $(\mathrm{H})$ & 15 \\
\hline & & dosulepin (dothiepin) (M) & 33 \\
\hline & & clomipramine $(\mathrm{H})$ & 15 \\
\hline & & nortriptyline $(\mathrm{H}, \mathrm{M})$ & 135 \\
\hline & & doxepin $(\mathrm{H})$ & 27 \\
\hline & & bupropion (L) & 3 \\
\hline & & mirtazapine (L) & 3 \\
\hline & \multirow[t]{7}{*}{ Antipsychotic } & quetiapine $(\mathrm{H}, \mathrm{M}, \mathrm{L})$ & 159 \\
\hline & & risperidone (L) & 138 \\
\hline & & prochlorperazine (M, L) & 126 \\
\hline & & haloperidol (M, L) & 93 \\
\hline & & olanzapine $(\mathrm{H}, \mathrm{M}, \mathrm{L})$ & 27 \\
\hline & & levomepromazine $(\mathrm{H})$ & 21 \\
\hline & & fluphenazine $(\mathrm{H}, \mathrm{L})$ & 3 \\
\hline & \multirow[t]{2}{*}{ Antiparkinson } & carbidopa (L) & 54 \\
\hline & & benztropine $(\mathrm{H})$ & 12 \\
\hline & \multirow[t]{8}{*}{ Anxiolytic (Benzodiazepine) } & temazepam (L) & 123 \\
\hline & & lorazepam (L) & 117 \\
\hline & & clonazepam (L) & 72 \\
\hline & & diazepam (L) & 57 \\
\hline & & triazolam (L) & 54 \\
\hline & & midazolam (L) & 24 \\
\hline & & oxazepam (L) & 12 \\
\hline & & alprazolam (H, L) & 9 \\
\hline & \multirow[t]{2}{*}{ Anticonvulsant } & valproic Acid (L) & 42 \\
\hline & & carbamazepine $(\mathrm{M}, \mathrm{L})$ & 33 \\
\hline & Antimanic & lithium (L) & 18 \\
\hline
\end{tabular}


Table 2 (continued)

\begin{tabular}{|c|c|c|c|}
\hline System & Therapeutic class & $\begin{array}{l}\text { Medicines with anticholinergic } \\
\text { activity }\end{array}$ & Frequency \\
\hline \multirow{11}{*}{$\begin{array}{l}\text { Gastrointestinal/ } \\
\text { genitourinary tract } \\
\text { system }\end{array}$} & Antispasmodic & atropine $(\mathrm{H})$ & 3 \\
\hline & Antidiarrheal & loperamide (M, L) & 45 \\
\hline & \multirow[t]{2}{*}{ Antacid } & ranitidine $(\mathrm{M}, \mathrm{L})$ & 42 \\
\hline & & cimetidine $(\mathrm{M}, \mathrm{L})$ & 3 \\
\hline & \multirow[t]{4}{*}{ Antihistamine } & loratadine (M, L) & 27 \\
\hline & & cetirizine $(\mathrm{M}, \mathrm{L})$ & 18 \\
\hline & & promethazine $(\mathrm{H})$ & 6 \\
\hline & & fexofenadine (M) & 3 \\
\hline & \multirow[t]{2}{*}{ Antiemetic } & metoclopramide (L) & 75 \\
\hline & & domperidone (L) & 63 \\
\hline & $\begin{array}{l}\text { Antimuscarinic (overactive } \\
\text { bladder agent) }\end{array}$ & oxybutynin (H, M) & 72 \\
\hline Muscle relaxant & & orphenadrine $(\mathrm{H})$ & 3 \\
\hline Immunosuppressant & & cyclosporine (L) & 6 \\
\hline \multirow[t]{5}{*}{ Opioid } & \multirow[t]{5}{*}{ Narcotic analgesic } & codeine $(\mathrm{M}, \mathrm{L})$ & 162 \\
\hline & & morphine (L) & 132 \\
\hline & & oxycodone (L) & 102 \\
\hline & & tramadol $(\mathrm{M}, \mathrm{L})$ & 30 \\
\hline & & methadone (M) & 15 \\
\hline \multirow[t]{7}{*}{ Miscellaneous } & Biguanides & metformin (L) & 480 \\
\hline & \multirow[t]{3}{*}{ Corticosteroid } & prednisone (L) & 171 \\
\hline & & dexamethasone (L) & 54 \\
\hline & & hydrocortisone (L) & 18 \\
\hline & Anti-gout & colchicine $(\mathrm{H}, \mathrm{L})$ & 36 \\
\hline & Antimetabolite & methotrexate (L) & 6 \\
\hline & COMT inhibitor & entacapone (L) & 3 \\
\hline
\end{tabular}

$n=69$, i.e. the total number of unique anticholinergic activity medicines captured in this study was adopted from one of our previous publications [49].

Anticholinergic activity is indicated by L (low), M (moderate) or H (high). ACE = Angiotensin-converting enzyme; SSRI = selective serotonin reuptake inhibitor; SNRI = serotonin-norepinephrine reuptake inhibitor; COMT = catechol-0-methyltransferase.

and nonlinear model buildings is depicted in online supplementary table S1 (see www. karger.com/doi/10.1159/000441718.

Table 2 summarises the frequencies of the most commonly prescribed medicines with anticholinergic activity identified from the study population. The anticholinergic activity of medicines is rated based on a published reference scale inferring high $(\mathrm{H})$, moderate $(\mathrm{M})$ and low (L) activity [49]. The most frequently used anticholinergic medicines are those for the treatment of cardiovascular and neurological disorders (table 2). The majority of the anticholinergic medicines identified had L anticholinergic activity (80\%), including furosemide, metoprolol, citalopram, metformin, warfarin, digoxin, isosorbide and diltiazem. $\mathrm{M}$ to $\mathrm{H}$ activity anticholinergic medicines included amitriptyline, quetiapine, nortriptyline, prochlorperazine, haloperidol and paroxetine. $\mathrm{L}, \mathrm{M}$ and $\mathrm{H}$ activity anticholinergic medicines were used for calculating the burden scores shown in table 2. Several anticholinergic activity medicines 
Table 3. Patient characteristics of interest influencing anticholinergic adverse events derived from the final (nonlinear) model

\begin{tabular}{ll} 
Factors & aOR $(95 \% \mathrm{CI})$ \\
\hline ACh burden & $3.21(1.23-5.81)$ \\
Non-ACM & $1.41(1.31-1.51)$ \\
Age & $1.08(1.05-1.10)$ \\
CCI & $0.84(0.78-0.95)$
\end{tabular}

ACh burden = Sum of total anticholinergic medicines; non-ACM = non-anticholinergic medicines; $\mathrm{CCI}=$ Charlson comorbidity index; $\mathrm{aOR}=$ adjusted odds ratio; $\mathrm{CI}=$ confidence interval.

available in the literature [49] were not applicable to the study population because either they are not available in New Zealand or they are funded by PHARMAC. A complete list of these medicines is outlined in online supplementary table S1.

The final model was a nonlinear model for predicting the probability of anticholinergictype events. Those patient characteristics that were found to be influential were ACh burden, non-ACM, age and CCI (table 3). The main patient characteristics was ACh burden (aOR of an anticholinergic event was 3.21, 95\% CI: 1.23-5.81, for those taking an average of 5 anticholinergic medicines compared to those taking $1 ; p<0.05$ ). It was a strong independent risk factor for developing an anticholinergic event. Other patient characteristics that were found to be significant included: non-ACM (aOR 1.41, 95\% CI: 1.31-1.51) and age (aOR 1.08, 95\% CI: 1.05-1.10). The CCI score was negatively associated with anticholinergic events (aOR 0.84, 95\% CI: 0.78-0.95). The influence of ACh burden was nonlinear within the logit domain, and the estimated maximum probability ( $E \max$ ) of an adverse anticholinergic event was 0.33 , i.e. with an infinite number of ACh burden medicines. The patient characteristics non-ACM, age and CCI were found to influence the baseline risk of an anticholinergic event. In addition, $\mathrm{CCI}$ and non-ACM were found to influence the maximum risk of an anticholinergic event associated with ACh burden (i.e. they affected $E \max$ ). None of the patient characteristics appeared to modify the effect of risk posed by ACh burden drugs.

\section{Discussion}

This study examined and explored the relationship between patient characteristics associated with anticholinergic drugs and anticholinergic-type adverse events in older people. The evidence from the current study clearly supports the existing literature that higher ACh burden, polypharmacy, age and multimorbidity are independent risk factors for adverse anticholinergic events in older people [2, 7, 51-53]. Findings from a recent cross-sectional study conducted in Finland involving 621 older individuals showed a strong association between ACh burden and anticholinergic events [54]. In a cohort study conducted by Rudolph et al. [31], higher anticholinergic risk scores were associated with an increased risk of both peripheral and central anticholinergic adverse effects in older people. The study compared two cohorts, one retrospective (132 patients) and one prospective (117 patients); in both cohorts, the risk of anticholinergic adverse events increased significantly with higher anticholinergic risk scores. Compared to the retrospective cohort, the prospective primary-care cohort reported dry mouth and constipation more frequently with an evidence of a doseresponse relationship [31]. 
Salahudeen et al.: The Influence of Patient Characteristics on Anticholinergic Events in Older People

A prospective study involving 532 older veterans reported that the prevalence of anticholinergic-type adverse events, such as dry mouth and constipation, was significantly higher in individuals using anticholinergic drugs [55]. The study highlighted that anticholinergictype adverse events are rare events $(0.8 \%)$, which mirrors the incidence rate of anticholinergic-type events in our study population (6.6\%) [55]. A study conducted in the USA involving 833 older home-care patients found that polypharmacy, a common occurrence in nursinghome residents, increases the risk of adverse drug events and hospitalisation [56]. Similarly, in our study, non-ACMs were associated with anticholinergic-type events both as an interaction with CCI and as an independent predictor.

In this study, we anticipated a positive association between comorbidities and anticholinergic events, adjusted for the total medicine burden; and the finding of a negative association is counterintuitive. We speculate that the burden of disease may take precedence over reporting of anticholinergic events. A new-user design excluding prevalent users (first-time users) may provide a clearer picture of the relationships between anticholinergic exposure and anticholinergic events, and this could be an area for future research.

The implications of this research could help to identify important patient characteristics that influence ACh burden. A reduction in ACh burden may result in improved short-term memory, confusion, behaviour and delirium together with an enhanced quality of life and daily functioning of older people [7, 28, 35]. In the context of quantification of ACh burden, drug dosage and muscarinic receptor-binding affinity are important to be considered. There is no globally accepted tool to quantify ACh burden for commonly prescribed medicines with known anticholinergic properties [10]. There is a need to improve the quantification of ACh burden. The model-based approaches presented in this study illustrate a potential direction for a nonlinear binding model to estimate the relative ACh burden of medicines.

One of the major strengths of this study was the use of population-level data and allowing the characteristics to be associated with the outcome of interest in a nonlinear model framework. The patient characteristics were derived from national health collections in real settings; hence, selection bias was negligible and makes our findings relevant to clinical practice. The findings reported in this study are generalisable as it represents the population of older people in New Zealand captured by the nationwide prescription database (Pharms). However, our findings need replication in other samples of diverse clinical settings for longer periods. The study also recognises important limitations of real data. The most significant concern is the potential of confounding by indication, something which is inherent to all pharmacoepidemiological studies.

The study did not account for all anticholinergic events contained in the national minimum dataset. Accurate documentation of anticholinergic-type adverse events relies on rigid clinical coding guidelines. Among the 2,248 individuals analysed in this study, only $2.1 \%$ had a coded diagnosis of delirium, which is considerably less compared to other database studies [40]. This may have underestimated the risk posed by ACh burden. Since causality was not assessed, we were unsure whether any of the events were caused by anticholinergics. A retrospective study design may be subject to residual confounding by unmeasured factors. The possibility of protopathic bias could not be entirely ruled out, as many older individuals receive anticholinergic medicines for the management of urinary symptoms. However, the choice of 3 diverse events, delirium, constipation and urinary retention, that arise commonly from anticholinergic activity medicines but less frequently from a single pathology was an attempt to decrease the risk of confounding factors associated with adverse events. This may cause an overestimate of the true association (positive confounding) or an underestimate of the association (negative confounding). It should be noted that this does not eliminate the risk. 


\section{Conclusion}

To our knowledge, this is the first study that examined population-level data in a nonlinear model framework to predict anticholinergic-type adverse events. This study evaluated the relationship between important patient characteristics and the occurrence of anticholinergic-type events. ACh burden was found to be an independent risk factor for predicting the probability of the anticholinergic-type events in older people. This study lends support to findings obtained from observational and experimental studies and reinforces the clinical significance of reviewing anticholinergic medicines in older people.

\section{Acknowledgments}

M.S.S. was funded by a Doctoral Scholarship from the University of Otago, Dunedin, New Zealand. The funder and sponsor had no role in the study design, data collection, analysis or interpretation. We would like to thank the Analytical Services, Ministry of Health of New Zealand, for supplying the prescription data extracted from the Pharms database.

\section{Disclosure Statement}

None of the authors have any conflicts of interest to declare.

\section{References}

1 Chew ML, Mulsant BH, Pollock BG, Lehman ME, Greenspan A, Mahmoud RA, Kirshner MA, Sorisio DA, Bies RR, Gharabawi G: Anticholinergic activity of 107 medications commonly used by older adults. J Am Geriatr Soc 2008;56:1333-1341.

2 Roe CM, Anderson MJ, Spivack B: Use of anticholinergic medications by older adults with dementia. J Am Geriatr Soc 2002;50:836-842.

-3 De Wilde S, Carey IM, Harris T, Richards N, Victor C, Hilton SR, Cook DG: Trends in potentially inappropriate prescribing amongst older UK primary care patients. Pharmacoepidemiol Drug Saf 2007;16:658-667.

-4 Narayan SW, Hilmer SN, Horsburgh S, Nishtala PS: Anticholinergic component of the drug burden index and the anticholinergic drug scale as measures of anticholinergic exposure in older people in New Zealand: a population-level study. Drugs Aging 2013;30:927-934.

5 Salahudeen MS, Hilmer SN, Nishtala PS: Comparison of anticholinergic risk scales and associations with adverse health outcomes in older people. J Am Geriatr Soc 2015;63:85-90.

6 Ganjavi H, Herrmann N, Rochon PA, Sharma P, Lee M, Cassel D, Freedman M, Black SE, Lanctot KL: Adverse drug events in cognitively impaired elderly patients. Dement Geriatr Cogn Disord 2007;23:395-400.

7 Feinberg M: The problems of anticholinergic adverse effects in older patients. Drugs Aging 1993;3:335-348.

-8 Montamat SC, Cusack BJ, Vestal RE: Management of drug therapy in the elderly. N Engl J Med 1989;321:303309.

-9 Tune L, Carr S, Hoag E, Cooper T: Anticholinergic effects of drugs commonly prescribed for the elderly: potential means for assessing risk of delirium. Am J Psychiatry 1992;149:1393-1394.

-10 Salahudeen MS, Duffull SB, Nishtala PS: Impact of anticholinergic discontinuation on cognitive outcomes in older people: a systematic review. Drugs Aging 2014;31:185-192.

11 Peters NL: Snipping the thread of life. Antimuscarinic side effects of medications in the elderly. Arch Intern Med 1989;149:2414-2420.

12 Tune LE: Anticholinergic effects of medication in elderly patients. J Clin Psychiatry 2001;62(suppl 21):11-14.

13 Tune LE, Egeli S: Acetylcholine and delirium. Dement Geriatr Cogn Disord 1999;10:342-344.

14 Karlsson I: Drugs that induce delirium. Dement Geriatr Cogn Disord 1999;10:412-415.

15 Nishtala PS, Fois RA, McLachlan AJ, Bell JS, Kelly PJ, Chen TF: Anticholinergic activity of commonly prescribed medications and neuropsychiatric adverse events in older people. J Clin Pharmacol 2009;49:1176-1184.

16 Remillard AJ: A pharmacoepidemiological evaluation of anticholinergic prescribing patterns in the elderly. Pharmacoepidemiol Drug Saf 1996;5:155-164. 


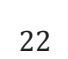

$-23$

$>24$

$-25$

26 Cao YJ, Mager DE, Simonsick EM, Hilmer SN, Ling SM, Windham BG, Crentsil V, Yasar S, Fried LP, Abernethy DR: Physical and cognitive performance and burden of anticholinergics, sedatives, and ace inhibitors in older women. Clin Pharmacol Ther 2008;83:422-429.

-27 Lechevallier-Michel N, Molimard M, Dartigues JF, Fabrigoule C, Fourrier-Reglat A: Drugs with anticholinergic properties and cognitive performance in the elderly: Results from the PAQUID study. Br J Clin Pharmacol 2005;59:143-151.

-28 Hilmer SN, Mager DE, Simonsick EM, Cao Y, Ling SM, Windham BG, Harris TB, Hanlon JT, Rubin SM, Shorr RI, Bauer DC, Abernethy DR: A drug burden index to define the functional burden of medications in older people. Arch Intern Med 2007;167:781-787.

-29 Murray AM, Levkoff SE, Wetle TT, Beckett L, Cleary PD, Schor JD, Lipsitz LA, Rowe JW, Evans DA: Acute delirium and functional decline in the hospitalized elderly patient. J Gerontol 1993;48:M181-M186.

-30 Bostock CV, Soiza RL, Mangoni AA: Association between prescribing of antimuscarinic drugs and antimuscarinic adverse effects in older people. Expert Rev Clin Pharmacol 2010;3:441-452.

-31 Rudolph JL, Salow MJ, Angelini MC, McGlinchey RE: The anticholinergic risk scale and anticholinergic adverse effects in older persons. Arch Intern Med 2008;168:508-513.

-32 Thomas C, Hestermann U, Kopitz J, Plaschke K, Oster P, Driessen M, Mundt C, Weisbrod M: Serum anticholinergic activity and cerebral cholinergic dysfunction: an EEG study in frail elderly with and without delirium. BMC Neurosci 2008;9:86.

-33 Schubert CC, Boustani M, Callahan CM, Perkins AJ, Carney CP, Fox C, Unverzagt F, Hui S, Hendrie HC: Comorbidity profile of dementia patients in primary care: are they sicker? J Am Geriatr Soc 2006;54:104-109.

-34 Mangoni AA, Munster BCV, Woodman RJ, Rooij SED: Measures of anticholinergic drug exposure, serum anticholinergic activity, and all-cause postdischarge mortality in older hospitalized patients with hip fractures. Am J Geriatr Psychiatry 2013;21:785-793.

-35 Tomida K, Takahashi N, Saito S, Maeno N, Iwamoto K, Yoshida K, Kimura H, Iidaka T, Ozaki N: Relationship of psychopathological symptoms and cognitive function to subjective quality of life in patients with chronic schizophrenia. Psychiatry Clin Neurosci 2010;64:62-69.

-36 Drimer T, Shahal B, Barak Y: Effects of discontinuation of long-term anticholinergic treatment in elderly schizophrenia patients. Int Clin Psychopharmacol 2004;19:27-29.

37 Molloy DW, Brooymans M: Anticholinergic medications and cognitive function in the elderly. J Clin Exp Gerontol 1988;10:89-98.

38 The Ministry of Health NZ: Health statistics and data sets; national collections of health and disability information. http://www.health.govt.nz/nz-health-statistics/national-collections-and-surveys/collections (accessed April 9, 2015).

39 Pharmaceutical management agency (PHARMAC). Update: New Zealand pharmaceutical schedule. Effective December 2010. http://www.PHARMAC.Govt.Nz/2010/11/18/su.Pdf (accessed April 9, 2015).

40 Ahmed S, Leurent B, Sampson EL: Risk factors for incident delirium among older people in acute hospital medical units: a systematic review and meta-analysis. Age Ageing 2014;43:326-333.

41 Siddiqi N, House AO, Holmes JD: Occurrence and outcome of delirium in medical in-patients: a systematic literature review. Age Ageing 2006;35:350-364.

42 Lieberman JA: Managing anticholinergic side effects. Prim Care Companion J Clin Psychiatry 2004;6:20-23.

43 Salzman C (ed): Clinical Geriatric Psychopharmacology, ed 4. Philadelphia, Lippincott, Williams and Wilkins, 2005.

44 Charlson ME, Pompei P, Ales KL, MacKenzie CR: A new method of classifying prognostic comorbidity in longitudinal studies: development and validation. J Chronic Dis 1987;40:373-383. 
45 Luukkanen MJ, Uusvaara J, Laurila JV, Strandberg TE, Raivio MM, Tilvis RS, Pitkälä KH: Anticholinergic drugs and their effects on delirium and mortality in the elderly. Dement Geriatr Cogn Dis Extra 2011;1:43-50.

-46 Nishtala PS, Narayan SW, Wang T, Hilmer SN: Associations of drug burden index with falls, general practitioner visits, and mortality in older people. Pharmacoepidemiol Drug Saf 2014;23:753-758.

47 Bostock CV, Soiza RL, Mangoni AA: Associations between different measures of anticholinergic drug exposure and Barthel Index in older hospitalized patients. Ther Adv Drug Saf 2013;4:235-245.

48 Duran CE, Azermai M, Vander Stichele RH: Systematic review of anticholinergic risk scales in older adults. Eur J Clin Pharmacol 2013;69:1485-1496.

-49 Salahudeen MS, Duffull SB, Nishtala PS: Anticholinergic burden quantified by anticholinergic risk scales and adverse outcomes in older people: a systematic review. BMC Geriatr 2015;15:31.

50 Hastie T: Generalized additive models; in Chambers JM, Hastie T (eds): Statistical Models in S. Pacific Grove, Wadsworth and Books/Cole Advanced Books \& Software, 1992, pp 249-307.

-51 Cancelli I, Beltrame M, D’Anna L, Gigli GL, Valente M: Drugs with anticholinergic properties: a potential risk factor for psychosis onset in Alzheimer's disease? Expert Opin Drug Saf 2009;8:549-557.

52 Gerretsen P, Pollock BG: Cognitive risks of anticholinergics in the elderly. Aging Health 2013;9:159-166.

53 Nishtala PS, Salahudeen MS: Temporal trends in polypharmacy and hyperpolypharmacy in older New Zealanders over a 9-year period: 2005-2013. Gerontology 2015;61:195-202.

54 Pasina L, Djade CD, Lucca U, Nobili A, Tettamanti M, Franchi C, Salerno F, Corrao S, Marengoni A, Iorio A, Marcucci M, Violi F, Mannucci PM: Association of anticholinergic burden with cognitive and functional status in a cohort of hospitalized elderly: comparison of the anticholinergic cognitive burden scale and anticholinergic risk scale: Results from the REPOSI study. Drugs Aging 2013;30:103-112.

55 Ness J, Hoth A, Barnett MJ, Shorr RI, Kaboli PJ: Anticholinergic medications in community-dwelling older veterans: Prevalence of anticholinergic symptoms, symptom burden, and adverse drug events. Am J Geriatr Pharmacother 2006;4:42-51.

56 Flaherty JH, Perry HM 3rd, Lynchard GS, Morley JE: Polypharmacy and hospitalization among older home care patients. J Gerontol A Biol Sci Med Sci 2000;55:M554-M559. 